\title{
An Analysis of the Changing Character of an Urban Street: A Case Study of the Galle Road, Colombo.
}

\author{
Nisha Fernando
}

\begin{abstract}
Analyses of the perceptible character of an environment are often vague and implicit. In urban contexts. visual elements are recognized as key attributes of the environmental character. This paper is based on a research study that investigated the environmental character within an explicit conceptual framework, which included several visual as well as nonvisual attributes. People's interactions with the environment. by means of various activities and settings, contribute significantly to the character of an urban space. Non-visual sensory elements, such as smell or sound, also contribute largely to the perception and evaluation of the ambience of an urban environment. Culture is the overarching concept and it plays an important role in the type of activity, the choice of settings, and the presence/absence of multi-sensory elements. Taking Galle Road, Colombo, a key urban street in Colombo and an example of the Sri Lankan urban cultural landscape, the present study considered activity systems. systems of settings. and multi-sensory elements as key attributes of the perceptible character. It focused specifically on a comparison of how the character changes at different points along the street, from the Lotus Road intersection at Galle Face to the Dehiwela Bridge. The methodology involved unobtrusive participant observations. place-centered behavioral mapping. systematic field notes, and photographic documentation. The findings showed that there are seven distinct sections of Galle Road that display different characters. Furthermore, these different sections, distinguished on the basis of how people use the street and the resultant multi-sensory qualities, do not coincide with various zones and divisions as outlined by a recent development proposal for the Galle Road.
\end{abstract}

Key Words: Perceptible character, activities, settings, multi-sensory clements

\section{Introduction}

Urban streets, in cultural contexts such as Sri Lanka, are vibrant public and social spaces. The public life generates a significant ambience in the environment of such urban streets. This ambience, or character, not only describes the physical environment per se, but it also brings a distinctive cultural identity to the overall urban landscape. Ambience, however, may not be the same at every point of the street; in other words, it may change from one place to another along the length of the street. These changing patterns need to be understood in any approach to design and implement urban planning proposals, so that they would be congruent with the existing physical fabric of the street as well its cultural idenitity.

Recent redevelopment proposals seem to consider Galle Road as a major urban street divided by a few major nodes or intersections created by arterial roads (CMRSP, 1998) (Fig. 1a \& 1b). These nodal divisions are further accentuated by various types of proposed land use. However, systematic observations (and day-to-day experiences) point to the fact that there are sub-ınits of a different calibre in the street. These sub-units are not necessarily distinguishable by the land use type or the physical fabric (such as the type and scale of the buildings), but by the types of activities, the people who engage in these activities and the resultant qualities they create. Since various combinations of these attributes creates a particular character in each sub-unit, it is necessary to discern such patterns in the character in order to understand the street as a whole. Understanding these changes in the character is also critical since people seem to perceive and use the street differently within the areas markedly different in character.

Another shortcoming of the development plans is the focus on only the visual characteristics of the street. This is not at all uncommon in new urban proposals in many parts of the world. However, it is critical to understand that the very nature of urban life closely relates to and operates on other senses as well, such as in auditory, olfactory, thermal, tactile, and kinesthetic modalities. People's activities and behavior are closely linked with such multi-sensory aspects of street life. 


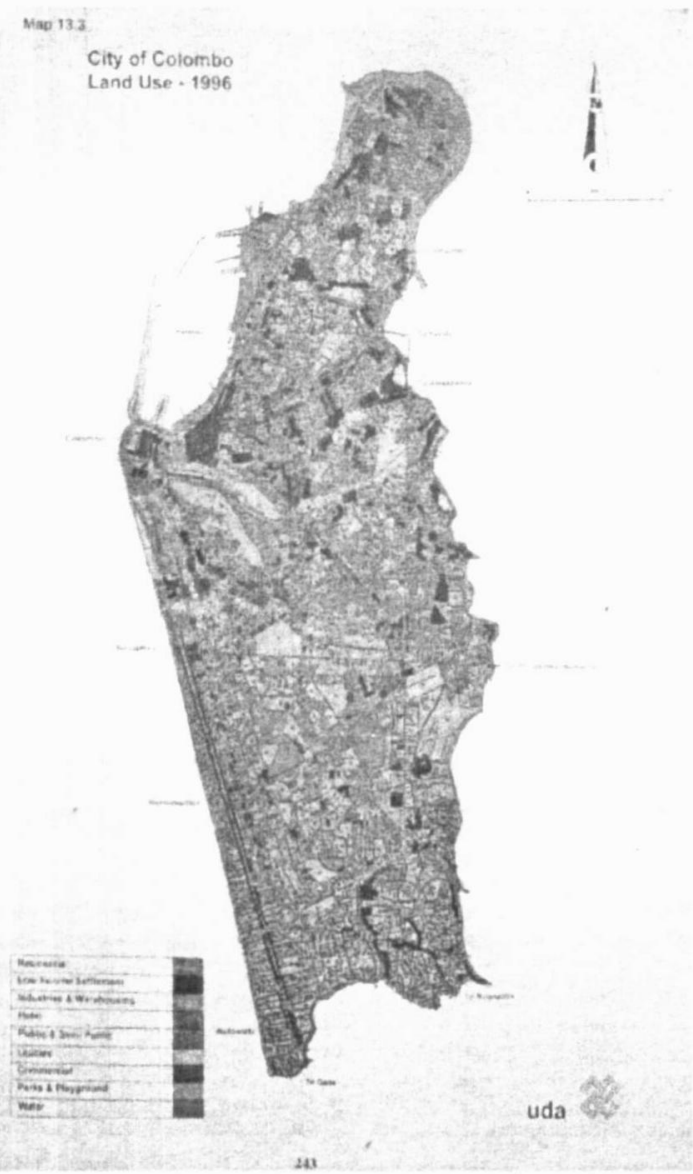

Fig. 1a: Existing land use patterns

(Note the fine grain of landuse)

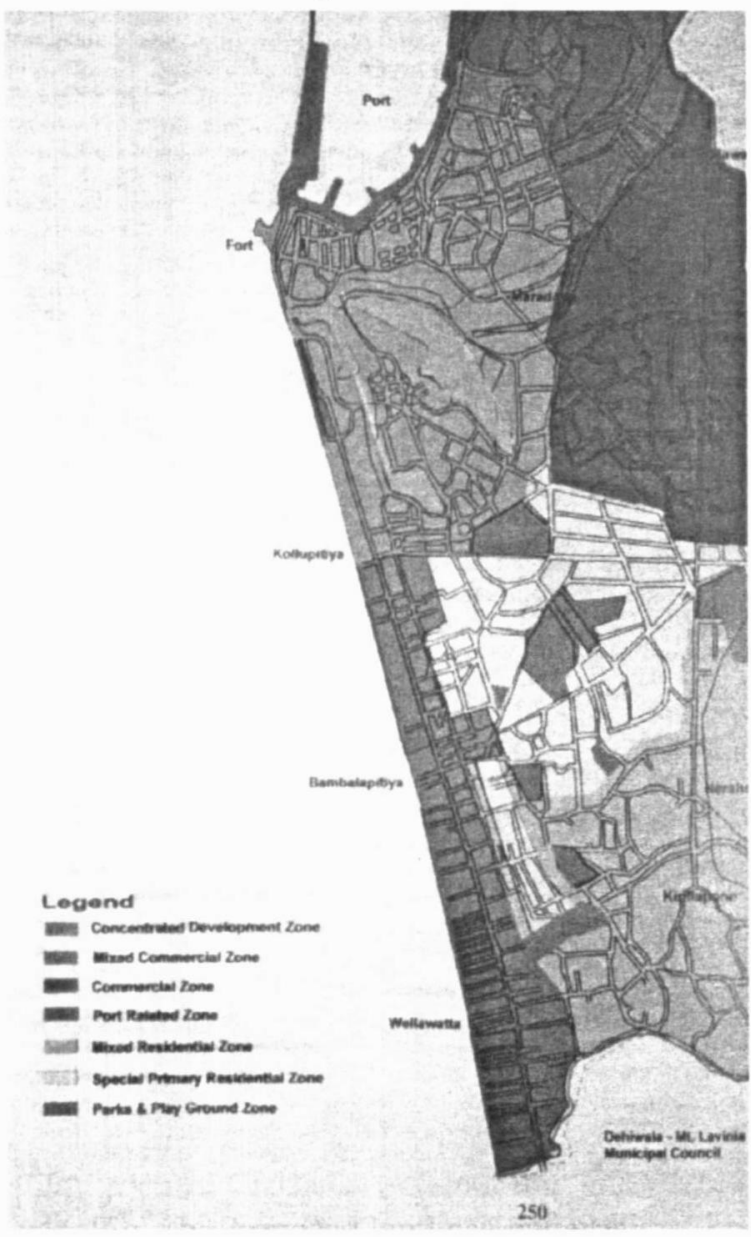

Fig. 1b: Proposed land use patterns

(Note the change in the grain of land use)
There are two reasons to study the changes in the character of the Galle Road. First, while the street is generally divided only by certain physical aspects such as landuse type and major intersections. actual activities of people seem to divide the street into rather diffrent sub-units. These sub-units display different ambiences or characters, and such distinctions must be systematically understood before any development proposal is executed. Second, the study of the perceptible character should not be limited to visual elements of the physical environment, but also to the various activity patterns and the resultant multi-sensory nature of the street.

Based on the above observations, an empirical research project was designed to examine the changing character of the Galle Road. The research study focused on the changes in the perception of the street from the beginning of the Galle Road (Lotus Road at Galle Face) to the Dehiwela Canal Bridge. The data was collected between June and August 1999 using multiple research methods and subsequently analyzed by a systematic content analysis and use of a GIS system.

\section{Research Questions}

Two research questions were formulated for this empirical study.

(1) Given that the character changes along the Galle Road creating sub-units, what specific attributes contribute to the character or ambience in each sub-unit?

(2) How do these sub-units coincide with the areas defined in the development proposals?

\section{The Environment-Behaviour Approach}

Since the character involves the physical aspects of the street as well as how people use the street for various activities and their behaviours, the research study employs an environment-behaviour(EB) approach to address the above mentioned research questions. The E-B concepts explicitly explain the various interactions between the users of the street and its physical environment.

\section{Galle Road as an "Urban Street"}

Urban streets are one category of urban environments defined rather loosely. Generally. streets are regarded as linear strips, roads or a pathways between two rows of buildings, the primary 
use of which is pedestrian and vehicular movement. In some contexts, as in most cities in the U.S., streets are conceived and treated as mere thoroughfares that run through the urban fabric. However, in other cultural contexts - such as in France, Italy, Egypt, South Africa, Taiwan, China, India, and Sri Lanka - urban streets are seen as public spaces vital to the social life. Here the interactions between people and the street are high and frequent. For the purpose of this research study, an urban street is first considered as a place type where the users interact with the physical environment of the street in various specific ways. It is conceptually defined as an entity of different systems of settings (Rapoport, 1990b; Liu, 1994) that support many different activities, carried out by different people at different times (Rapoport, 1990b). In the latter view, Galle Road is a particular type of cultural landscape that contains specific systems of settings that accommodate a variety of activities.

\section{Definition of "Character" or "Ambience"}

The character or ambience of any environment is another obscure and vague term. Descriptions of the character of physical environments often limit to visual elements such as forms, shapes, colors, aesthetics, and so on. Furthermore, only the physical fabric of urban environments- generally considered to comprise of buildings (Rapoport, 1977; 1993) - is used to describe the "urban" character. For instance, cities are identified to have a "historical" character, when the buildings reflect certain architectural or building styles from the past. Venturi, Scott Brown \& Izenour (1998) suggest that colorful signs and eye-catching objects are important for the "symbolic" character of Las Vegas. The character is also related to several different serial views of English small towns (Cullen, 1961). However, visual aspects not always prominent or significant in perception of cities. Rapoport (1977; 1992a; 1993) points out that the very first experience one may get of streets in India and bazaars in the Middle-East is generally through smells of food and incense, both non-visual characteristics. A study by Pred (1963) shows that some urban streets in Chicago were distinguishable from one another on differences in smell and sounds. These sensory characteristics resulted from when different ethnic groups occupying the streets engaging in activities such as cooking, smoking, and playing music. In Sri Lanka, sounds of drums and horns played during rituals at the Temple of the Tooth in Kandy create a rather unique ambience in the surrounding streets. In a different street in Kandy, the type of music and drums fixed, emerging from the Kataragama Kovil creates yet another ambience. All the examples show that non-visual experiences are as significant as, and can even be more critical than, visual experiences in perceiving the character of an urban environment.

The above study by Pred (1963) also suggests that the type of activity, where they take place, who is involved, and so on are also critical in identifying the character of a street. The smells of food and sounds of jazz music on the 47th street in Chicago is a direct result of the activity choice of AfricanAmericans, and therefore can be linked to their cultural aspects (Pred, 1963). In a section of Devon Street in Chicago (well known for its authentic Indian shops and restaurants), the smells of Indian food and sounds of Hindi music filter out of the restaurants and shops, creating a rather distinctive ambience. This section can be compared with an immedaitely adjacent section of the street, where a Russian community has established their own shops, restaurants and retails stores. The differences in various sounds and smells are very clear between these two areas. In addition, the density of people standing, sitting, strolling, chatting and socializing is higher in the Russian section than in the Indian section of the street. Pedestrians of each community also add a different character that is identifiable from different clothing patterns, dress styles, jewelry, accessories worn and carried, language, and so on.

As much as activities and multi-sensory experiences create the character, where such activities occur also contributes to the character of a street. Sometimes a particular activity can take place in more than one type of setting. For instance, personal observations of a street in the East Village, New York City, showed that some people socialize either sitting in an open cafe, on a ledge of a building, on benches in a mini-park, standing in front of a newspaper stand, on entrance steps of a shop, in a corner of the street, or even in the middle of a busy sidewalk. Liu (1994) supplements this observation by showing how a single street in Taiwan had numerous different settings. It is clear, then, that settings also contribute to distinctive differences, and therefore to the character, of streets. Such settings, like activities, can be specific to a culture, and thus, are culture-specific.

Type of settings are also important to the ambience of a street not only because of the activities taking place in them, but also because they describe its physical environment, or what it is "made of." Rapoport $(1977 ; 1990 b)$ explains that any physical environment can be dismantled into fixed, semi- 
and non-fixed components. These components can be numerous, but they provide an explicit measurements to any environment. Fixed components of a street may include "fixed" elements such as buildings, sidewalks, roads, curbs, steps, railings, light and telephone poles, telephone booths, and so on. Semi-fixed components may include various "movable" objects such as street furniture, plants, billboards, notice boards, signs, flags, banners, food carts, boxes, trash cans, and so on. Non-fixed components are people, animals, and vehicles. Particular arrangements of these elements can depict a distinctive character that is specific to a cultural context. Some examples are the enclosed streets with colorful tent roofs along with the sacks of colorful grains, spices and fruits in Morocco; over crowded streets in Indian cities accommodating fast moving vehicles side by side with cows; and streams of people on bicycles and scooters among the skyscrapers of Singapore. These arrangements are results of very specific ways people interacting with the streets, based on their cultural background. Fixed, semi-fixed and non-fixed elements are important components of the cultural landscape of a street and therefore are vital cultural expressions.
The attributes of the perceptible character of an urban street, then, can be summarized as the activities, the settings in which these activities take place, and the multi-sensory characteristics created as a result of the activities. What specific activities take place, who is engaged in such activities, what specific physical elements contribute to the activities, and what specific sensory experiences are created are related to the cultural aspects of the users.

\section{The Conceptual Framework}

As discussed earlier, the attributes of the perceptible character of a street are products of the specific culture. Rapoport (1977) shows that culture is a very broad concept and too abstract to be useful to study the interactions between people and the environment. For example, the concept activity systems can be empirically observed, but not the abstract concept of culture.
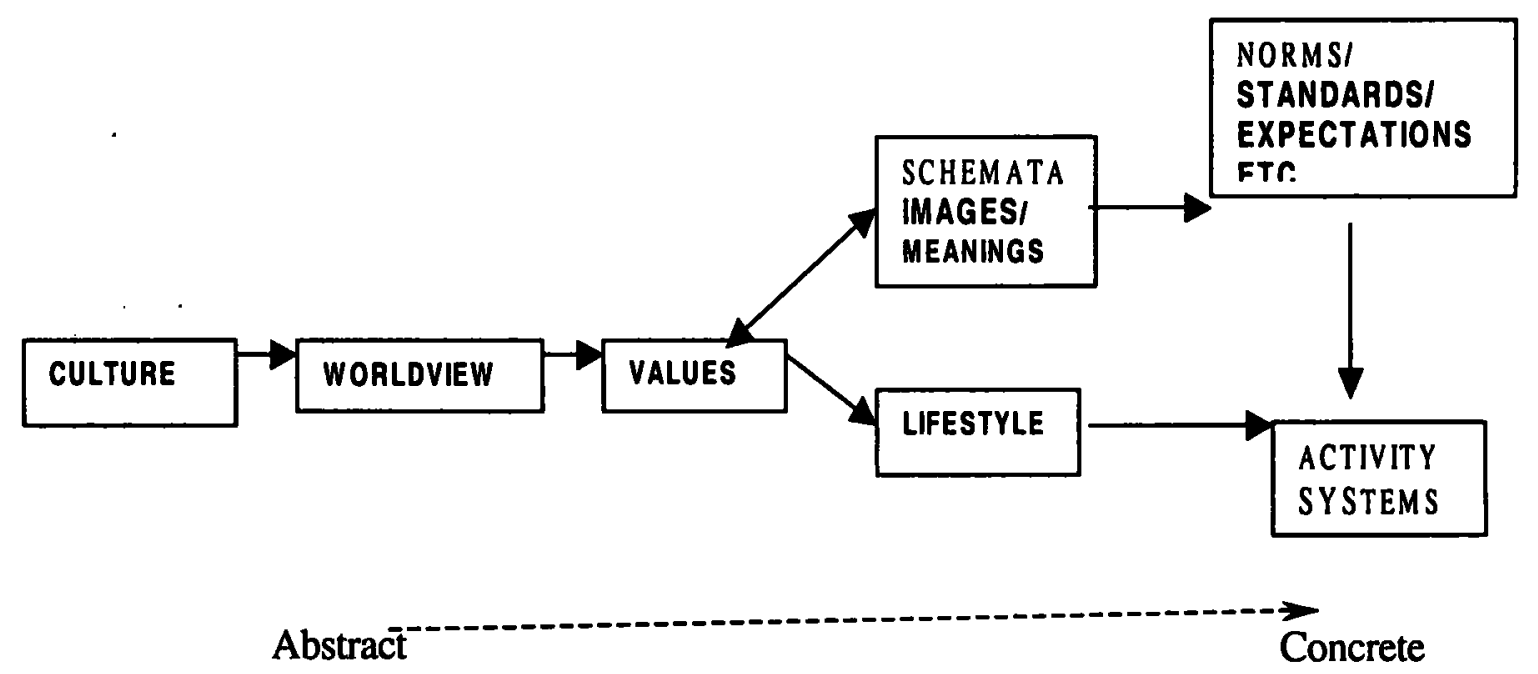

Fig. 1a: Concept of Culture Dismantled (Rapoport, 1977. 1993, in press)

\section{Noticeable Differences}

Given that there are specific attributes of the perceptible character, how can one identify it? What makes one character different from another? Rapoport (1977; 1982a; 1990a; 1992a) suggests that some elements become more noticeable than others, resulting in "noticeable differences." Certain elements can stand out against the back ground, and this difference is what is noticed. Rapoport (1990a) also points out that it is necessary to understand the relationships between elements rather than elements themselves and the juxtaposition of various elements lead to noticeable differences. Potential noticeable differences in the attributes of the character as they apply to each different sub-unit of Galle Road are as below: 


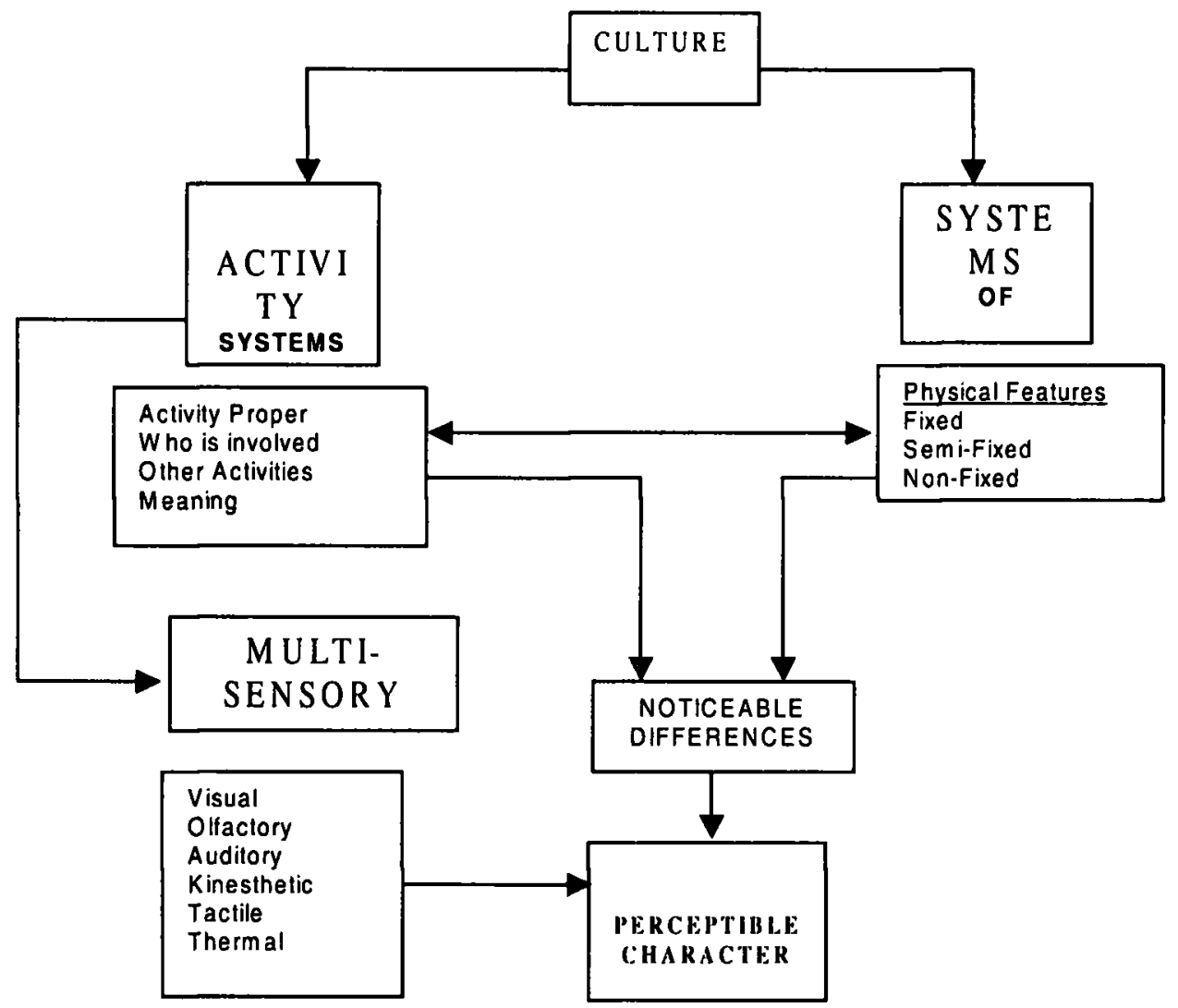

Fig. 2b: Conceptual Framework of Attributes of Perceptible Character

(Also Linking Culture to Perceptible Character)

\section{(1) Activity Systems:}

What is the particular activity?

What are the other related activities?

How are they different from the rest of the street?

\section{(2) Systems of Settings:}

Where does each activity take place?

What fixed, semi-fixed and non-fixed elements contribute to each activity?

How are they different from the rest of the street?

(3) Multi-Sensory Modalities:

Forms, shapes \& colors

Smells

Sounds

Textures

Kinesthetics/Movement Patterns

Air temperature /Light \& Shade

\section{Methodology}

\section{(1) "Eyeballing"/ Preliminary Observations}

This method enabled identification of the sections of the Galle Road that are seemingly different from each other. By comparing casual observations, distinct sections of the street were recognized. This was followed by the identification of the approximate "boundaries" of each section.

\section{(2) Unobtrusive Participant Observation}

As data could be affected if the users in streets became aware of the researcher's purpose, unobtrusive observations were carried out while collecting data, with a high degree of participation in the ongoing activities in the street. These participant observations were documented on a pre-set map of each street segment. Several codes were used for the activity types, fixed, semi-fixed and non-fixed elements, and multi-sensory elements present.

\section{(3) Behavior Mapping - Place-Centered Maps}

Place-centered behavior mapping was a useful method to document where activities are taking place, and what activities are present in a given location. Place-centered maps also showed where activities were concentrated or where activities were absent. The patterns of activities and settings were identified after comparing several maps of a particular location. 


\section{(4) Archival Research and On-Site Documentation}

Data was collected in the forms of in-field and off-field notes and diagrams and sketches, photographic slides.

\section{Data Analysis}

From the data gathered, seven sections could be identified as distinctively different from each other. The physical boundaries of these sections were crossroads and a canal; however, they were merely approximate boundaries of where activities would start or stop, and were by no means definite determinants of the activities.

(1) Section A:Lotus Road Intersection to Muhandiram's Road

(2) Section B: St. Michael's Road to Alfred House Avenue

(3) Section C: Alfred House Avenue to De Vos Avenue

(4) Section D: De Vos Avenue to Vajira Road

(5) Section E: Vajira Road to Sinsapa Road

(6) Section F: Sinsapa Road to 33rd Lane

(7) Section G: 33rd Lane to Dehiwela Canal Bridge

(1) SECTION A (Lotus Road Intersection to Muhandiram's Road):

\section{Land Use and Scale of Buildings}

This section is the beginning point of the Galle Road. A few commercial high-rise buildings and a large, open public space by the sea dominate street at the beginning. As a result, this side of the street is lined with a continuous strip of high walls. The street displays a more enclosed nature with medium-rise buildings lined on either side of the street as it continues further south.

\section{Activities and Settings}

As various security regulations have inhibited public activities around the Galle Face area, the street is fairly empty of people or activities all day and night, except for security personnel, motorists and an occasional pedestrian. The building facades along with high walls do not at all respond to the street, adding to the barrenness of the street (Fig. 3).

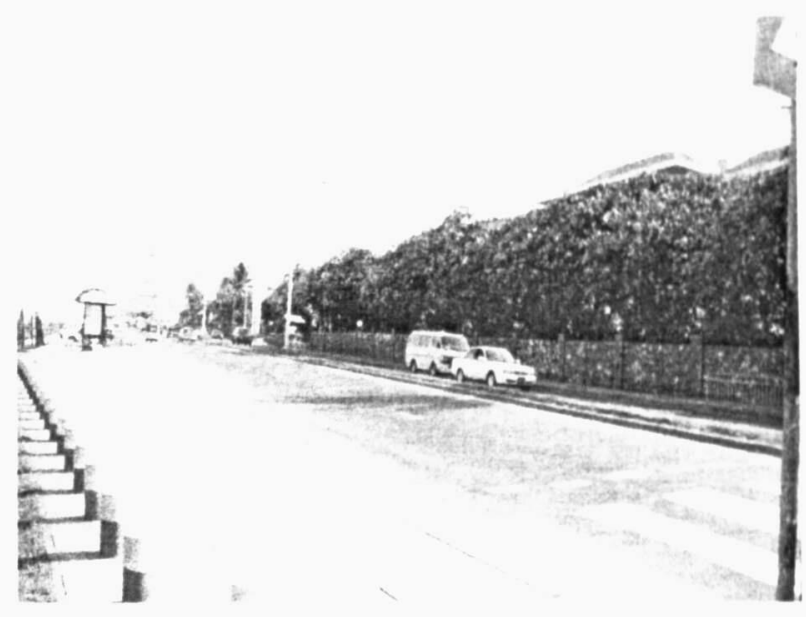

Fig. 3: Blank walls of buildings and "empty" sidewalks on the street

The next area of Section $\mathrm{A}$ does not accommodate that many activities either. Even though the land use pattern is different from the previous section (i.e. from hotels to offices and commercial buildings), the buildings respond very similarly to the street. Most buildings either have high walls or chain links in front and are set back from the street. Moreover, almost all commercial buildings except small retail shops have closed doors as a result of air-conditioned interiors. There are some activities in front of the retail shops, but since there are very few such shops in this section of the street, the activities are very few in number. In addition, there are physical (security) barricades on the sidewalk at certain points to inhibit pedestrian activities. As a collective result, the diversity of activities is minimal. The activities recorded more than three times were walking, standing, waiting for someone and shopping. Less frequent activities were street vending and socializing.

Considering the few activities and the settings that accommodate the activities, Section A seems far from being a lively public space. Yet, the very absence of many pedestrian activities generates an "empty" character. This "empty" character is reinforced by the heavy security control and imposed rules on pedestrian-related activities.

\section{Multi-Sensory Elements}

Because it is open to the sea, the beginning of Section A is characterized with the constant smell of salt. Also, as the area is open to the sea and the sun without any buildings or shady trees, the temperature or shadow patterns change scarcely during daytime. As the street continues southward, the difference in the temperature is immediate; the 
tall buildings on either side some shade patterns during the day. During mornings, the east side of the street is under shade while the west side is under the exposure of the sun, and in late afternoons, this pattern of light and shade switches over. There were no distinctive smells of food present. Since the traffic has been reduced for security purposes, the area has low levels of traffic noise. The commercial stores facing the street have very few and insiginificant display windows without any bold signage or eye-catching displays. The street is flat and linear there is no significant changes in the movement patterns.

(2) SECTION B: (St. Michael's Road to Alfred House Avenue)

\section{Land Use and Scale of Buildings}

Although the street continues to be enclosed with buildings, the type of land use changes significantly in this section. Most buildings are 4-6 storeys tall, housing various retail and commercial activities. There are no notable government buildings that require high security or surveillance. Most retail stores place items outside the store, almost on the street, along with a large number of signs, notices and display boards. The major node of Colpetty links an upscale commercial and business district (Navam Mawatha), a prominent Buddhist temple complex (Gangaramaya), two large shopping malls, a busy railway station and several schools to the Galle Road, attracting a large influx of people regularly to this particular section of the street.

\section{Activities and Settings}

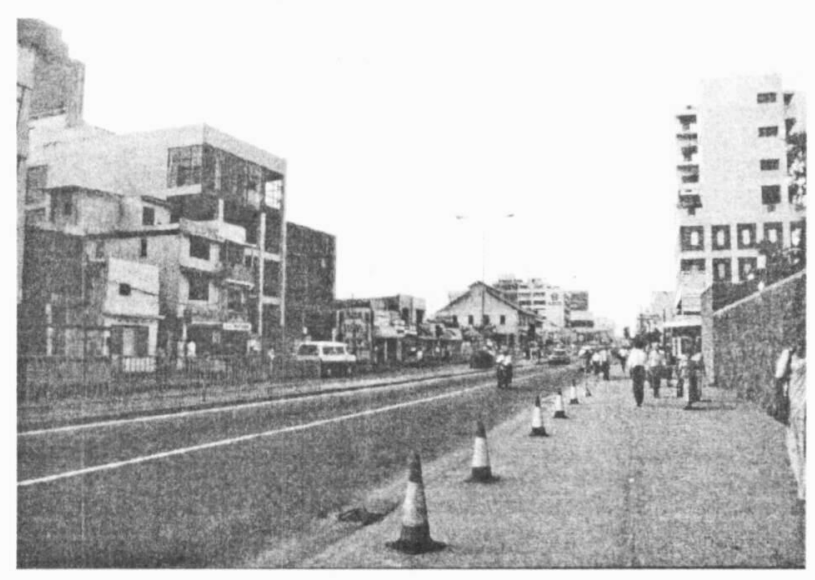

Fig. 4: Unresponding building facades and lack of activities in the street rather askewed.
In general, Section B has a higher rate of activities than the previous section (Fig.4). The activities and settings that stand out and are more noticeable than others were street vending, shopping, walking, standing, waiting for buses, and waiting for someone. Less frequent activities included panhandling and socializing. Fixed elements of the settings were the sidewalks, entrance steps to a building, railings, and bus shelters; semi-fixed elements included temporary kiosks, mobile carts, tables, stools, display signs and boards, baskets and cardboard boxes; non-fixed elements were people (moderate number), vehicles (moderate to large number), and a very few animals (crows).

The most significant difference between Sections A and $\mathrm{B}$ was not only the higher number of activities and settings, but also the peculiar organizations of activities (i.e. activity systems). For example, a person waiting for a bus was also watching a housewife buying fruits from a street vendor; yet another person was smoking and reading a newspaper over someone's shoulder under a eave of a shop; and a third person was chatting with a friend while getting her umbrella mended by a cobbler on the front steps of a shop. The street space in this section provides opportunities for manipulating various fixed, semi-fixed and non-fixed elements and accommodating these diverse activities, creating activity systems. Such systems are a vital part of the perceived character of the street.

\section{Multi-Sensory Elements}

Street vendors' display of colorful clothes, umbrellas, fruits, and vegetables bring an immediate experience to the eye. In addition, open eateries and restaurants continuously emit different smells of food. For example, a restaurant on one side of the street and a small way-side eatery with snacks and drinks on the other side provide very different food smells and add to two discrete olfactory characters in either side of the street. Amplified sounds of the vendors selling lottery tickets are common, as well as music emitting from various shops. The traffic noise, including horn blowing, shouts from bus conductors, and police whistles, is also prominent especially during certain hours. Because of the varying scales of the buildings, the play of shadow and light is similar to the latter part of the Section A. There is no noteworthy pattern of kinesthetics, except for the area around the Colpetty junction where street vendors and their paraphernalia had occupied most of the sidewalk space and movement along the street is made to be 
(3) SECTION C:

(Alfred House Avenue to De Vos Avenue)

\section{Land Use and Scale of Buildings}

This section is similar in land use to Section B - the types of uses are primarily commercial and retail. There is, however, a notably higher rate of retail activities rather uniformly distributed throughout the section. The smaller buildings are used as various retail stores such as restaurants, eateries, snack bars, pharmacies, shoe shops, clothing stores, taverns, casinos, a market and a bank. A popular up-scale shopping mall cum theatre complex is also located in this section. The street is also close to a busy railway station, a mosque, a university complex and a prime residential area. A striking feature is the consistent use of the sidewalk for a very large number of activities. The sidewalk is as wide as any other section of the street, but due to many activities and the types of settings they accommodate, it seems to project a compact and dense character.

\section{Activities and Settings}

Like the previous section, there is a major intersection in this area and this node (i.e. Bambalapitiya) creates a prominent hub of activities. The intersecting road as well as the railway station bring in a variety of people to this node. In addition, the diversity of retail and commercial stores attract a large number of people as well.

The most frequent activities are street vending (fruits, vegetables, used books, clothes, plastic wristwatches, cosmetic items, newspapers, etc), walking, shopping, socializing, waiting for a bus, waiting for someone, people- watching, idling and panhandling. Other activities include cleaning the street and eating or drinking (Fig. 5). The fixed features include the sidewalk, railings, folded iron doors of the shops, roof overhangs, and bus shelters; semi-fixed elements include cardboard boxes, makeshift tables, stools, mobile lottery and gram carts, umbrellas, garbage cans, various signs and display boards, posters, baskets and so on; and non-fixed features are people (a very large number), cars, buses, auto rickshaws ("three-wheelers"), bicycles, dogs, cats and crows.

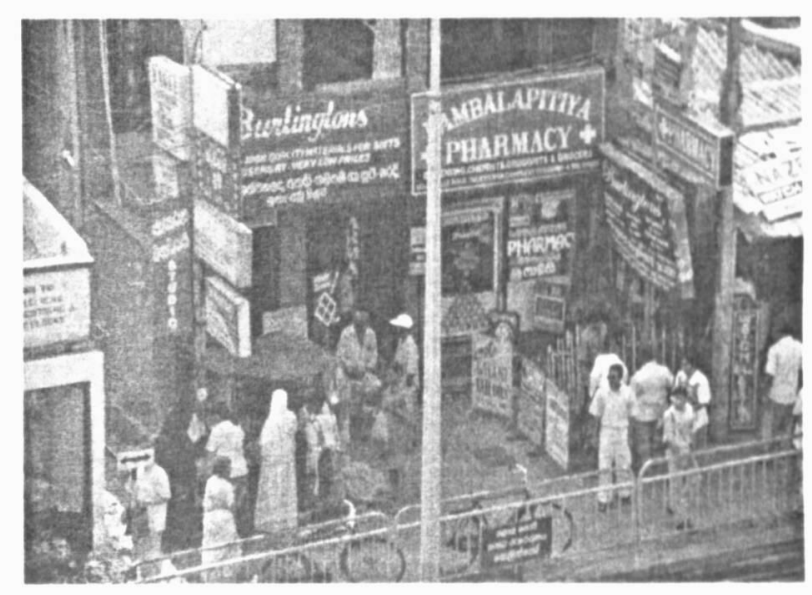

Fig. 5: Use of many fixed, semi-fixed, and non-fixed elements and presence of several activities

A very noticeable element is the type of settings accommodating all the activities - almost every possible space is utilized for some form of activity, and some times more than one activity, making this section a very complex, vibrant activity hub.

\section{Multi-Sensory Elements}

As a result of many different activities, this section seems to provide a variety of sensory experiences. First, many retail shops adorn brightly colored broads, signs, and posters. To attract pedestrian customers, shops display their ware and goods at shopfronts in the street. The density and closeness of such shops and street vendors selling an assortment of things, most rather colorful (e.g. fruits. vegetables, hair accessories, toys, etc), creates an attractive visual quality. As a result of fruit, and vegetable stalls, snack carts, and open eateries, a range of olfactory experiences is always present. Most retail shops and street vendors light incense sticks and this results in another distinctive smell. The public market's garbage collection area results in a foul smell as well.

Snack bars and eateries constantly play loud music and these sounds mix in with the sounds of lottery sellers, street performers, shouts of street vendors and constant traffic noise. Aggreagted activities in the steeet make it impossible for one to walk in a linear manner; rather, one is "forced" to meander around street vendors and the numerous activity areas. These kinesthetic patterns on the street were best observed from the top of a tall building. Light and shade patterns contribute to a somewhat different experience. Due to many roof overhangs and more temporary canopies, many shops and restaurants along the street provide additional shade 
for activities. As a result of these elements, the experiences of hot and cool temperatures are sudden and add to the shifting experiences of the section. In summary, this section of the Galle Road provides a variety of sensory experiences that add to the differences noticed in the street. The concurrent presence of many sensory modalities induces a unique character to this section, and results in an ambience strikingly different to the preceding sections of the street.

(4) SECTION D: (De Vos Avenue to Vajira Road)

\section{Land Use and Scale of Buildings}

A very prominent Hindu temple complex exists on the eastern side. The building is set further back from the street, but the surrounding open compound is immediately next to the street. The compound runs along almost entire length of the eastern side of Section $D$, but is visually hidden from the street by a 20 -foot high wall, with an equally tall iron gate. However, several small commercial establishments and retail stores, that tie with the activities of the temple, open out to the street. In fact these shops are the only suggestions to a layperson of the presence of the temple beyond the wall.On the other side of the street some medium and small commercial buildings line the street and they show no notable difference to those in Section C. However, this section does not portray the same levels of intensity and diversity of the activities as in Section C. As a result, this area of the street is relatively empty of people and a bustling character.

\section{Activities and Settings}

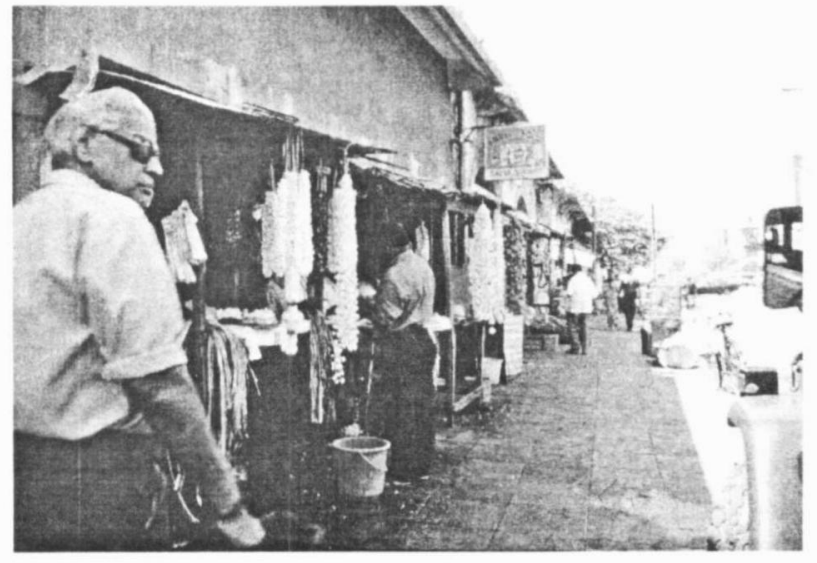

Fig. 6: Multi-sensory elements along the temple wall

Activities of on the eastern side of the street are imprinted by the presence of the temple complex and therefore the area reflects a religious atmosphere.
Although mobile street vending is not prominent in this section, almost all retail shops display goods at shopfronts and some kiosks are set up along the wall facing the street itself. These shops and kiosks sell flowers garlands, incense, betel leaves, fruits and other items used regularly for Hindu rituals (Fig. 6). There are also shops with colorful clothes and glittering jewllery, and restaurants that emit ethnic food smells, bringing in rather unique ambience to this section. The settings are mainly shop fronts and the sidewalk, with only a few pedestrians. The western side has a few retail and commercial activities not linked with the temple complex. They are limited to walking, street vending, shoe repairing.

\section{Multi-Sensory Elements}

Although the activities are comparatively low in number, the overall sensory experience in this section, especially in the eastern side, is dramatically different from Section C. When one walks into this section, the most noticeable characteristic is that the cocacophony of the sounds from Section C suddenly being subdued. Instead, the only consistent sound is the Tamil music flowing out of the shops. Colors of the flowers, fruits, signs and clothes also provide a significant character in this section. As a result of these colors, this section clearly stands out among the rest of the street. The other striking difference is in the distinctive smells in this section, especially from the incense, flowers and the food.

\section{(5) SECTION E: (Vajira Road to Sinsapa Road)}

\section{Land Use and Scale of Buildings}

The primary land use type of this section, again, is commercial/mixed commercial. The marked difference of this section, however, is that the building fabric is not very vertical and therefore the street is not enclosed. Since tall buildings are scarce and are scattered among many small-scale buildings, there is a sense of semi-openness in the entire section. There is also more vegetation present than the previous sections. Mixed land use types include residential flats, two small-scale hotels, gas stations, bookstores, clothing stores, furniture stores, restaurants, photo studios and retail grocery stores.

\section{Activities and Settings}

Apart from being less enclosed, this section is similar to the Section D because of the lack of activities. Although there are many commercial 
activities, they are generally limited to interiors of the buildings. There are small pockets of street activities but as they are rather unevenly distributed along the street, they cannot be accounted for to reflect a continuous stream of settings seen in other sections(Fig. 7). Overall, the few activities on the street give an appearance of an urban residential area. The immediate area beyond this section of the street is in fact primarily residential and the users this section of the street comprise of these residents from these neighborhoods. In addition, this particular section is in between two major road intersections (the intersection in Section $\mathrm{C}$ and another in Section F) where activities are much more concentrated. As a result, there is a decreased sense of public attractions in this area of the street. One of the most noticeable aspects of this section was that during early afternoons (between 1-3), there were no people whatsoever on the road. This indicates that the main users of the street are residents. However, the limited number of activity types does bring a certain quiet ambience to this section.

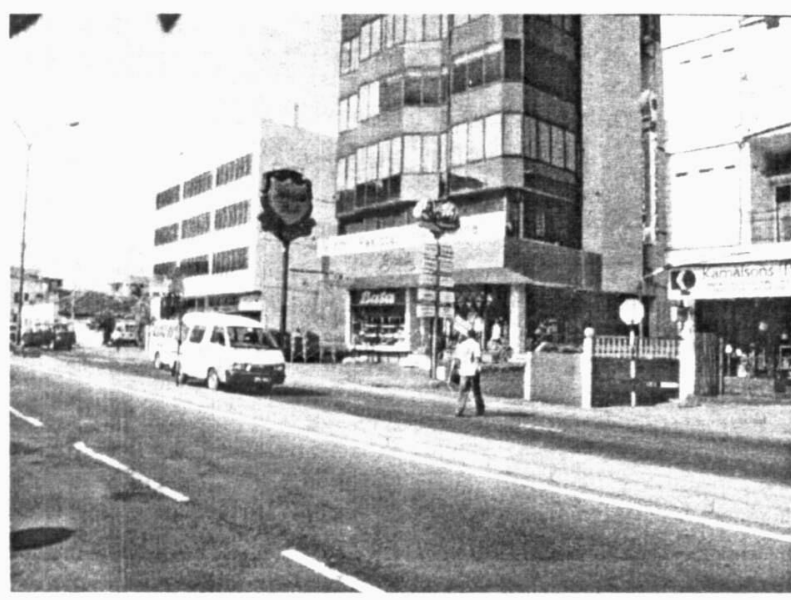

Fig. 7: Scattered buildings along the edge

\section{Multi-Sensory Elements}

As a direct result of the lack of activities, the multi-sensory experiences are rather different in this section. There are neither particularly colorful elements in the limited number of settings, nor there are any signs and roadside notice boards with eye-catching images, as there are only a few pedestrians. Probably the only visual difference is the presence of more greenery and vegetation. Since this area is in between two main intersections, there is a lesser degree of traffic congestion and as a result, the traffic noise is rather low. Interestingly, the Hindu temple present in this section is very different to that of Section D. Although the high outer wall shows a street facade very similar to that of the temple in
Section D, there are no retail activities outside the wall indicating the existence of a temple. As a result, there are no colors, smells or sounds that are identifiable as a part of the temple and related religious activities.

\section{(6) SECTION F: (Sinsapa Road to 33rd Lane)}

\section{Land Use and Scale of Buildings}

Rather different from Section E, this section closely resembles Section C. The whole section is vibrant with the presence of many people and numerous activities. The significant difference, however, is the scale of the buildings, which is notably smaller than that of Section C. The majority of the buildings are 2-3 storeys high, while there are some single storey buildings as well. There is also a major node because of the intersection an arterial road. This road introduce many people to this section of the street throughout the day; hence, the increased number of activities. There is a larger variety of building types as well. Most buildings are small-scale retail stores selling a variety of goods such as groceries. vegetables and fruits, hardware, books, clothes, jewelry, cosmetics, antiques, shoes and so on (Fig. 8). The public market is a relatively small space attracting a large number of people, while there are also numerous very small shops and kiosks (some under the overhead bridge).

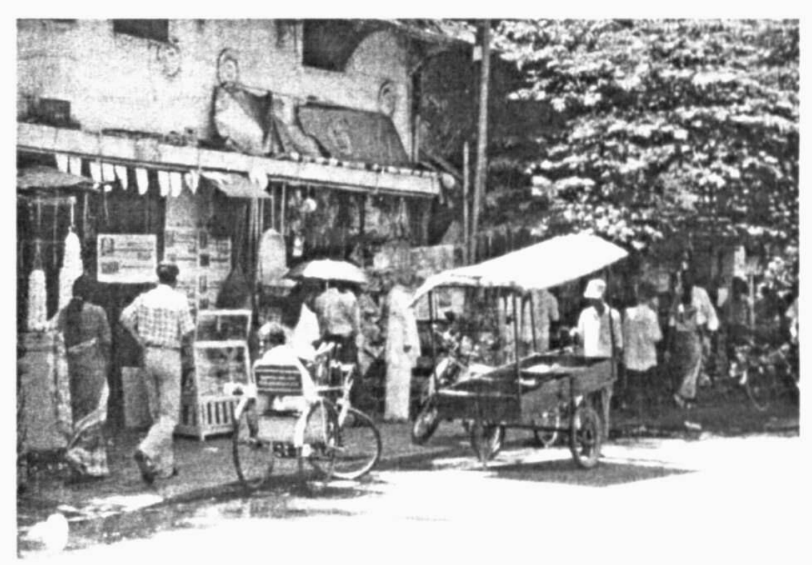

Fig. 8: Activity hub and several settings

\section{Activities and Settings}

This section is another intensive activity hub. There is a large number of activities on the street and these activities, like in Section C, are diverse in type. Since the majority of the population in this area is Tamil, the activities and the types of elements in the settings create a character that is very different from other sections (except Section D), thus making 
the area very distinctive. The most noticeable and frequent activities are street vending (a variety of goods), shopping, window-shopping, walking, standing, socializing, waiting for a bus, and cleaning. The settings are numerous as well: fixed features include open shops, steps, kiosks, the over-head bridge and sidewalks; semi-fixed features include boxes, makeshift furniture, signs and posters, banners, mobile carts, baskets, gunny bags and sacks, and hanging lamps; non-fixed features are people, bicycles, auto rickshaws, buses and cars, dogs, cats, crows, pigeons and cows. The particular combinations of these environmental features are very unique in this section.

\section{Multi-Sensory Elements}

As a result of many activities and various different settings, the sensory experience in this section is rather strong. The people themselves add the visual appearance of the street, with brightly colored shiny clothes, females with flowers in their hair and heavy jewellery. Many open stalls with colorful vegetables and fruits also contribute to the overall visual character. Many restaurants and shops play Tamil music almost continuously. Vendors consistently shout over each other's voice to attract people. Traffic noise increases again, along with shouts of bus conductors, car horns, bicycle bells and police whistles. Olfactory experiences are particularly striking. There is a range of specific smells, from highly perfumed incense and oil from the shops to strong smells of vegetables and fruits to foul smells of fish and trash. Pedestrian movement in this section takes a rather non-linear fdue to the many activities taking place in the sidewalk. It is very similar to the immediate experiences in the Section C, except for differences in the juxtaposition and intensity of various sensory modes. There are also noticeable differences in the language and type of music overheard while in the street.

\section{(7) SECTION G:}

\section{(33rd Lane to Dehiwela Canal Bridge)}

\section{Land Use and Scale of Buildings}

This section changes from Section F rather significantly. While their scale remains the same, buildings are rather scattered along the street. There is a significant amount of vegetation in the street as well as in between buildings, making the streetscape punctuated and built spaces separated. The land use pattern varies considerably among these buildings from a theater to a church, Buddhist temple, gas stations, some retail shops and two small-scale hotels.
In between there are many residences. Most buildings have medium-height parapet walls. The few commercial buildings are air conditioned, and there are very little activities on the street. Overall, there is a mixed presence of commercial and residential character in the physical environment.

\section{Activities and Settings}

Reflecting the mixed-residential nature of this section, there is a lesser number of activities on the street. As a result, there is a very low degree of utilizing the physical elements. Especially, the number of semi-fixed elements present in this section is very low. The few activities that occur somewhat regularly in this section are walking/strolling, waiting for a bus and mobile vending (on bicycles and carts).

In contrast to the Sections $\mathrm{C}$ and $\mathrm{F}$, this area is devoid of street activities that are continuous through out the day (except vehicular traffic). It represents a typical residential area with limited and inconsistent activities on the street. The absence of any retail activities and street vending is probably due to the distance from the activity hub of the Section F. Towards the southern end of the street, even beyond the Dehiwela Canal Bridge, the ambience is clearly that of a residential area, without many prominent retail activities. Even the Buddhist temple, which is at the corner of the bridge, does not seem to induce any particular street activities.

\section{Multi-Sensory Elements}

Since the number of activities is significantly less, this section does not have an aggregation of noticeable sensory qualities. There are occasional sounds of mobile vendors ringing bicycle bells or of ice cream sellers blowing horns. There are no distinctive smells at all and this fact makes this section very different from all other sections of the street discussed earlier.

\section{Use of a GIS System in Data Analysis}

A map with the approximate boundaries of these seven sections was overlaid with another map showing the boundaries of the proposed developments by CMRSP (1998) by using a GIS system. The results showed that the boundaries of the two separate maps did not coincide at all. The spatial analysis also showed that the major intersections that defined the landuse boundaries in the development proposals were in fact within the different sections defined by how people use the street and by the different patterns of ambience in this study. 


\section{Discussion And Conclusions}

The analysis of activities, settings and multisensory modalities in each section of the street showed that the perceptible character varies rather significantly from one section to another. The analysis also showed that activities, settings or multi-sensory elements alone do not represent the perceptible character. For example, Section C and Section F displayed very distinctive characters, though both had very similar types of activities and settings; notable multi-sensory characteristics in Section F brought about a very different character. On the other hand, the very absence of activities and the lesser degree of sensory experiences produced a particular perceptible character ( i.e. "almost empty" character) in Section A.

The buildings were critical not only because of their physical appearance or the scale, but also because of their role in accommodating (or not accommodating) many different activities. Many open stores provided shopfronts for street vending; steps and entrances for socializing and people watching. Such activities could be hardly observed in front of the air-conditioned, introverted buildings. Even if the building was of large scale, if there were open shops and other facilities at the street level the number of activities increased rather remarkably. This proves that the physical scale and the actual number of storeys of a building do not necessarily contribute to the overall character of the street. The critical factor seems to be the provision of activities through the building and positive responses to the ongoing activities on the street. Also, the findings point out that the olfactory experiences were increased as a direct result of open restaurants, shops, and markets, while auditory elements (e.g. music from the shops) certainly added to the overall ambience. Therefore, rather than the scale of the buildings, their permeability at the street level seemed to be an important contribution to the overall character of the street.

In addition to the fixed elements, semi-fixed elements and non-fixed elements also were critical attributes. The lack of such elements in Section A brings a certain ambience, while the presence and the intensity of them in Sections $C \& F$ brings other types of ambiences. These elements are as critical as buildings themselves in the street environment, especially understanding it as an urban social space.
It was also clear from the analysis that multisensory experiences contribute to the perceptible character significantly. For example, the ethnic character of the Section D was more related to the colors, sounds and smells than the specific activities. Section $\mathrm{F}$ also produced a character largely discernible by different sensory elements. The comparative study of the different sections showed otherwise: visual. olfactory, auditory, tactile, and even thermal experiences individually contributed to the character of the sections.

The analysis confirmed the initial hypothesis that the street can be divided into sub-units that have different perceptible characters. It also reinforced the conceptual approach that used activities, settings and multi-sensory elements as essential attributes of perceptible character. This answers the first research question. Proposed land use boundaries, as explained earlier, take into account the areas between major intersections and implement development activities accordingly. But the actual activity hubs do not stretch from one intersection to another; rather, they have different boundaries and each intersection is included within the activity hub. More importantly, it is vital to understand the existing activity pattern and the "social grain" in each section of the street before making any development decisions. For example, if development plans proposed upscale commercial activities for both Section C and Section D, it may result in drastic changes to the existing use and the public perception of the Galle Road. It is important to remind ourselves that the Galle Road is not merely a commercial space, but rather a widely and consistently used urban public space that reflects a social and cultural identity. It certainly needs no imposed and dramatic physical alterations but perhaps mere opportunities for gradual modifications.

\section{References:}

CMRSP - Colombo Metropolitan Regional Structure Plan. (1998). Colombo, Sri Lanka: Urban Development Authority. Ministry of Housing and Urban Development.

Cullen, Gordon. (1961). Townscape. London: The Architectural Press.

Fernando, Nisha. (1999). A Preliminary Analysis of Urban Streets in Sri Lanka as Vernacular Cultural Landscapes. Unpublished Term Paper. Department of Architecture. University of Wisconsin-Milwaukee. 
Liu, Chi-Wen. (1994). From Old Town to New City: A Study of Behavior Settings and Meanings of Streets in Taiwan. Unpublished Ph.D. Dissertation. Department of Architecture, University of Wisconsin-Milwaukee.

Pred, Allan. (1963). Business thoroughfares as expressions of urban Negro culture. Economic Geography. July. 39: 217 233.

Rapoport, Amos. (1977). Human Aspects of Urban Form. New York: Pergamon Press.

Rapoport, Amos. (1829). On the perceptual separation of pedestrians and motorists. In H.C. Foot, A.J. Chapman, \& F.M Wade (Eds.), Road Safety (Research and Practice). New York: Praeger.

Rapoport, Amos. (1990a). History and Precedent in Environmental Design. New York: Plenum.

Rapoport, Amos. (1990b). Systems of activities and systems of settings. In S. Kent (Ed.), Domestic Architecture and the Use of Space. Cambridge, U.K.: Cambridge University Press.

Rapoport, Amos. (1992a). On regions and regionalism. In N.C. Markowich, W.F.E. Preiser, \& F.G. Strum. (Eds.), Pueblo Style and Regional Architecture. New York: Van Nostrand Reinhold.

Rapoport, Amos. (1992b). On cultural landscapes. Traditional Dwellings and Settlements Review. Vol.3. No.2 (Spring). 33-47.

Rapoport, Amos. (1993). Cross-Cultural Studies and Urban Form. College Park, Maryland: University of Maryland.

Venturi, Robert., Scott Brown, Denise., \& Izenour, Steven. (1998). (Revised Edition) Learning From Las Vegas. Cambridge, MA: MIT Press.

Nisha Fernando is currently reading for her $\mathrm{Ph}$.D.in Architecture at the University of Wisconsin-Milwaukee, USA. Her main research focus is on culture-specificity of the environment-behavior interactions and her current work involves an analysis of selected urban streets in the New York City. This paper presents a summarized version of a research project in Sri Lanka conducted in 1999. 